\title{
Dickkopf-1 is involved in BMP9-induced osteoblast differentiation of C3H10T1/2 mesenchymal stem cells
}

\author{
Liangbo Lin, Quanhe Qiu, Nian Zhou, Wen Dong, Jieliang Shen, Wei Jiang, Ji Fang, Jie Hao E Zhenming Hu ${ }^{*}$ \\ Department of Orthopaedic Surgery, the First Affiliated Hospital of Chongqing Medical University, Chongqing 400016, China
}

\begin{abstract}
Bone morphogenetic protein 9 (BMP9) is a potent inducer of osteogenic differentiation of mesenchymal stem cells. The Wnt antagonist Dickkopf-1 (Dkk1) is involved in skeletal development and bone remodeling. Here, we investigated the role of Dkk1 in BMP9-induced osteogenic differentiation of MSCs. We found that overexpression of BMP9 induced Dkk1 expression in a dose-dependent manner, which was reduced by the P38 inhibitor SB203580 but not the ERK inhibitor PD98059. Moreover, Dkk1 dramatically decreased not only BMP9-induced alkaline phosphatase (ALP) activity but also the expression of osteocalcin (OCN) and osteopontin (OPN) and matrix mineralization of $\mathrm{C} 3 \mathrm{H} 10 \mathrm{~T} 1 / 2$ cells. Furthermore, exogenous Dkk1 expression inhibited Wnt/ $\beta$-catenin signaling induced by BMP9. Our findings indicate that Dkk1 negatively regulates BMP9-induced osteogenic differentiation through inhibition of the Wnt/ $\beta$-catenin pathway and it could be used to optimize the therapeutic use of BMP9 and for bone tissue engineering. [BMB Reports 2016; 49(3): 179-184]
\end{abstract}

\section{INTRODUCTION}

Bone morphogenetic proteins (BMPs) are members of the transforming growth factor $\beta$ superfamily and in human consists of at least 15 BMPs (1-3). BMPs play an important role in stem cell proliferation and osteogenic differentiation(3, 4). Bone morphogenetic protein 9 (BMP9) was first identified in the developing mouse liver, and is one of the most potent BMPs in terms of promoting osteogenic differentiation of mesenchymal stem cells (MSCs) $(1,4,5)$. Both TGF $\beta$ type I and type II receptors are essential for BMPs to initiate their signaling, leading to gene regulation through both Smad and MAPK pathways $(1,6)$. BMPs regulate a distinct set of downstream

*Corresponding author. Tel: +86-13628326226; Fax: +86-238901 1217; E-mail: spinecenter_hu@126.com

http://dx.doi.org/10.5483/BMBRep.2016.49.3.206

Received 11 October 2015, Revised 10 November 2015, Accepted 15 December 2015

Keywords: Bone morphogenetic protein 9, Dickkopf-1, Mesenchymal stem cells, Osteoblast differentiation, Wnt/ $\beta$-catenin signaling targets that likely play a role in osteoinduction, including Runx2 and Osterix (7). Knocking out BMP signaling result in various skeletal and extraskeletal abnormalities during embryonic development and organogenesis in the mouse (8).

$\mathrm{Wnt} / \beta$-catenin signaling also plays an important role in skeletal development and osteoblast differentiation (9-11). Wnts are a family of secreted proteins that bind to their cognate receptor frizzled $(\mathrm{Fz})$ and low-density lipoprotein receptor related proteins (LRP) 5/6 co-receptors, and activate various signaling pathways, including the canonical $W n t / \beta$-catenin pathway. Activation of the canonical Wnt pathway leads to $\beta$-catenin accumulation in the cytoplasm and translocation into the nucleus, where it associates with Tcf/Lef transcription factors to regulate the transcription of target genes (10). Numerous factors, including LRP5/6 (12) and $\beta$-catenin (13), participate in the $\mathrm{Wnt} / \beta$-catenin signaling cascade associates to the bone mass, suggests that the canonical Wnt/ $\beta$-catenin signaling plays an important role in osteogenic differentiation and bone formation.

Dickkopf-1(Dkk1), a Wnt inhibitor, binds to LRP5/6 and Kremen $1 / 2$, which inhibits canonical Wnt signal and so prevents $\beta$-catenin degradation and osteoblast differentiation (14). Dkk1 is crucial for skeletal development, and regulation of limb development and head induction (15). Endogenous Dkk1 expression is detected primarily in osteoblasts and osteocytes (16), and is involved in bone formation and bone disease. Dkk1 heterozygous mutant $(\mathrm{Dkk} 1+/-)$ mice exhibit increased bone mass (17), whereas overexpression of Dkk1 is associated with osteolytic metastatic bone disease in prostate carcinoma (18) and multiple myeloma (19). Collectively, these results suggest that Dkk1 may function as a potent negative regulator of bone mass.

Both BMPs and the Wnt/ $\beta$-catenin signaling pathway are important for inducing osteogenic differentiation of MSCs, and recent studies have indicated the existence of cross-talk between BMP9 and Wnt signaling (20-22), which is important for the regulation of osteoblast differentiation. Although $\mathrm{Wnt} / \beta$ catenin signaling is crucial for BMP9-mediated induction of osteogenic differentiation, the role of the Wnt inhibitor Dkk1 in the crosstalk between $\mathrm{Wnt} / \beta$-catenin and BMP9 signaling remains to be fully elucidated.

In this study, we sought to determine whether Dkk1 plays an important role in BMP9-induced osteogenic differentiation of 


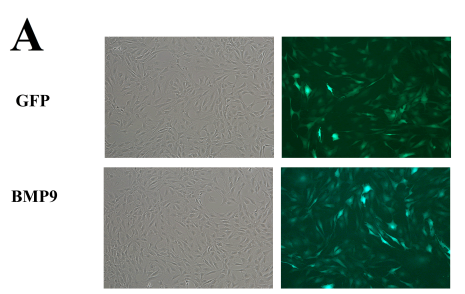

B

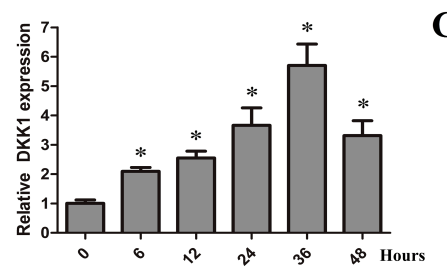

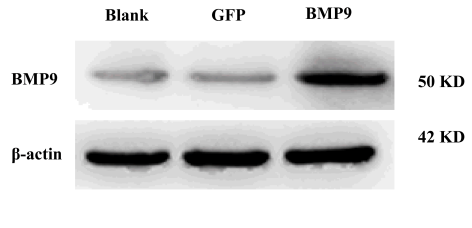

C

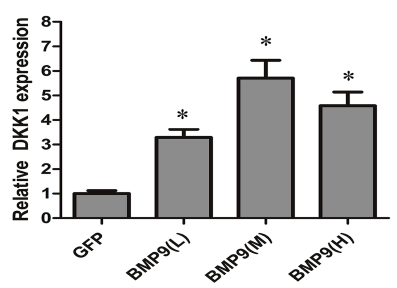

Fig. 1. Effect of BMP9 on Dkk1 expression. (A) Efficient transduction of C3H10T1/2 cells by AdBMP9. Cells were infected with AdBMP9 or AdGFP. Fluorescence images were recorded at $24 \mathrm{~h}$ after infection. Western blotting showed AdBMP9-mediated expression of BMP9 (MOls $=10)$ at $24 \mathrm{~h}$ after infection. (B) Time course of the effect of BMP9 on Dkk1 expression as assessed by qRT-PCR. mRNA was isolated from C $3 \mathrm{H} 10 \mathrm{~T} 1 / 2$ cells treated with AdBMP9 at $6,12,24,36$, and $48 \mathrm{~h}$ after infection. $* \mathrm{P}<0.01$ compared with $0 \mathrm{~h}(\mathrm{C})$ Dose-dependent effects of BMP9 on Dkk1 expression as assessed by qRT-PCR. C3H10T1/2 cells were coinfected with AdBMP9 at MOls of 20 (high), 10 (medium), and 5 (low) or GFP. *P $<0.01$ compared with GFP.
MSCs. We found that Dkk1 expression was upregulated by BMP9 in a dose-dependent manner in $\mathrm{C} 3 \mathrm{H} 10 \mathrm{~T} 1 / 2$ cells. Inhibition of P38 by SB203580 reduced the expression level of Dkk1 induced by BMP9. BMP9-induced osteogenic differentiation was inhibited by overexpression of Dkk1. Moreover, Dkk1 blocked BMP9-induced Wnt/B-catenin signaling activity. Taken together, our results suggest that BMP9 upregulates Dkk1 expression via the P38 MAPK pathway, and Dkk1 plays an important role in the negative feedback control of BMP9-induced osteoblast differentiation in MSCs through inhibition of the $\mathrm{Wnt} / \beta$-catenin pathway.

\section{RESULTS}

\section{Overexpression of BMP9 upregulates Dkk1 expression in C3H10T1/2 cells}

To examine the role of Dkk1 on BMP9-induced osteogenic differentiation of MSCs, We first investigated the potential link between the expression of Dkk1 and BMP9 signaling. C3H10T1/2 cells were infected with AdGFP and AdBMP9 (Fig. 1 left). By Western blotting, we found that the endogenous expression of BMP9 was very low but detectable and was upregulated at $24 \mathrm{~h}$ after infection. Moreover, AdGFP did not influence the expression of BMP9 in C3H10T1/2 cells (Fig. 1 right). C3H10T1/2 cells were infected with AdGFP or AdBMP9 (at low, medium, and high doses). Dkk1 expression was detected after $6 \mathrm{~h}$, and peaked at 5.7-fold at $36 \mathrm{~h}$ after treatment with BMP9, as assessed by qRT-PCR (Fig. 1B). Moreover, overexpression of BMP9 induced Dkk1 expression in a dose-dependent manner (Fig. 1C). These results suggested that BMP9 directly upregulated the expression of Dkk1.

\section{MAPK-P38 signaling is required for BMP9-induced Dkk1 expression}

Besides Smad transcription factors, studies have suggested that mitogen-activated protein kinases (MAPKs), such as P38 and

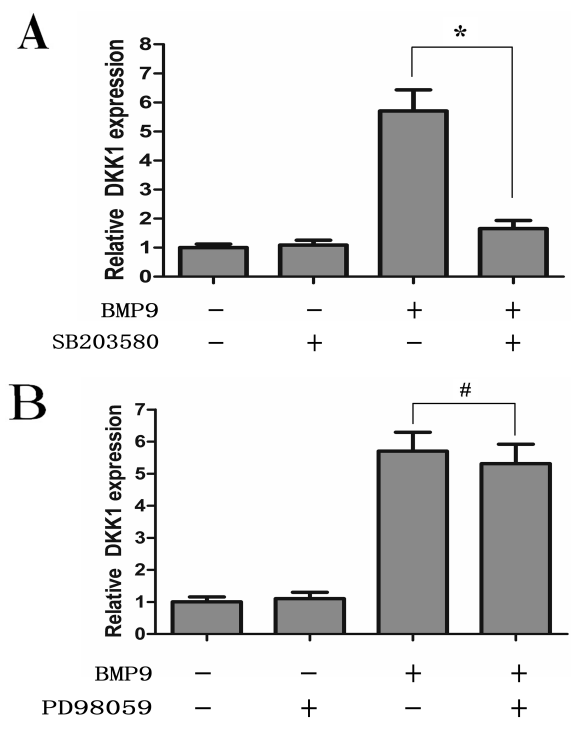

Fig. 2. Effects of MAPK signaling on Dkk1 expression induced by BMP9. mRNA was isolated from C3H10T1/2 cells pretreated with SB203580 $(10 \mu \mathrm{M})$ or PD98059 $(25 \mu \mathrm{M})$ for $1 \mathrm{~h}$ prior to treatment with AdBMP9 $(\mathrm{MOI}=10)$ for $36 \mathrm{~h}$. (A) Effect of the P38 inhibitor SB203580 on Dkk1 expression as assessed by qRT-PCR. Upregulation of Dkk1 expression by BMP9 treatment was blocked by SB202190 pretreatment. ${ }^{*} \mathrm{P}<0.01$. (B) Effect of the ERK1/2 inhibitor PD98059 on Dkk1 expression as assessed by qRT-PCR. " P $>0.05$.

ERK1/2, are involved in transmitting BMP signals intracellularly $(23,24)$. To determine the roles of P38 and ERK $1 / 2$ in BMP9-induced Dkk1 expression, C3H10T1/2 cells were exposed to AdBMP9 in the presence of SB203580 or PD98059, which are selective inhibitors of p38 and ERK1/2 activation, respectively. Upregulation of Dkk1 expression by BMP9 treatment was blocked by SB203580, as assessed by qRT-PCR (Fig. 2A). However, the ERK inhibitor PD98059 did not affect 
A

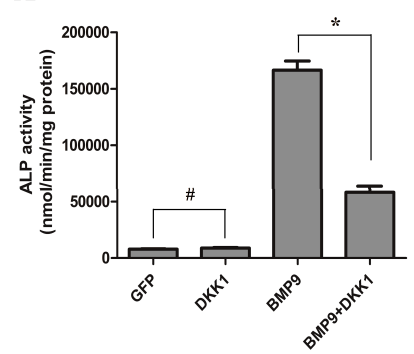

C

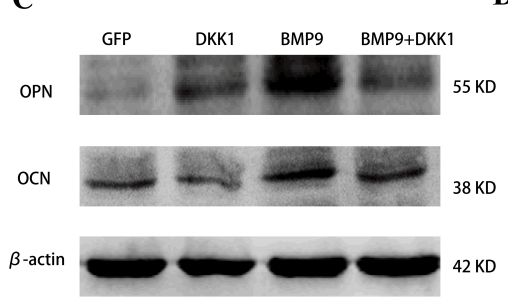

B
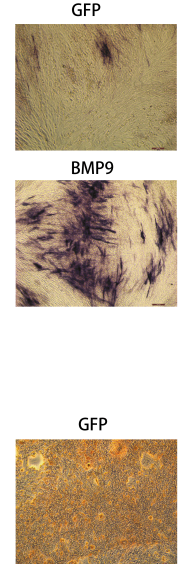

BMP9

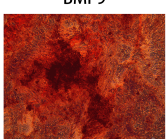

DKK1

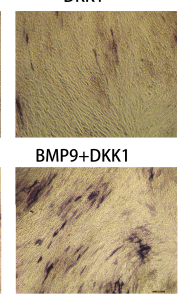

DKK1

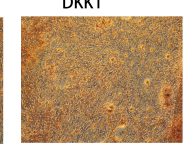

BMP9+DKK1

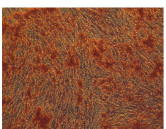

Fig. 3. Effect of exogenous Dkk1 expression on BMP9-induced osteogenic differentiation of MSCs. (A) Effect of Dkk1 on BMP9-induced ALP activities in $\mathrm{C} 3 \mathrm{H} 10 \mathrm{~T} 1 / 2$ cells. ${ }^{\#} \mathrm{P}>0.05,{ }^{*} \mathrm{P}<$ 0.01. (B) ALP histochemical staining showed the effect of Dkk1 on BMP9induced ALP activities in C3H10T1/2 cells. (C) Western blotting showed the effect of Dkk1 on the expression of OCN and OPN induced by BMP9 in C3H10T1/2 cells. (D) Alizarin Red S staining results showed the effect of Dkk1 on mineralization induced by BMP9 in C3H10T1/2 cells.
BMP9-induced Dkk1 expression (Fig. 2B). These findings showed that BMP9-induced Dkk1 expression might be mediated by the MAPK-P38 pathway.

\section{Dkk1 inhibits BMP9-induced osteogenic differentiation of MSCs}

We next assessed the effect of exogenous Dkk1 on BMP9-induced osteogenic differentiation of MSCs. C3H10T1/2 cells were coinfected with AdGFP, AdBMP9, and/or AdDkk1. ALP activity is an early well-established osteogenic marker of MSCs, which peaks at $\sim 7$ days after BMP9 stimulation (20). Exogenous Dkk1 alone did not induce ALP activity over the GFP control (Fig. 3A and B). As expected, BMP9 induced a significant increase in ALP activity, which was dramatically decreased by Dkk1 (Fig. 3A and B). We further evaluated the effect of Dkk1 on the late stage of BMP9-induced osteogenic differentiation. Osteocalcin (OCN), osteopontin (OPN), and matrix mineralization are well-established markers of late-stage osteogenic differentiation $(1,25)$. OCN and OPN were detected at 9 days after treatment by Western blotting, and mineralization was detected at 14 days. Consistent with the ALP results, Dkk1 treatment significantly decreased BMP9-induced expression of OCN and OPN (Fig. 3C) and matrix mineralization (Fig. 3D). Taken together, the above results strongly suggested that Dkk1 inhibited both the early and late stages of BMP9-induced osteogenic differentiation of MSCs.

\section{Exogenous Dkk1 expression inhibits Wnt/ $\beta$-catenin signaling induced by BMP9}

The canonical $\mathrm{Wnt} / \beta$-catenin signaling pathway is crucial for induction by BMP9 of osteogenic differentiation and bone formation (20-22). Therefore, we examined the contribution of $W n t / \beta$-catenin signaling to the inhibition of BMP9-induced os-
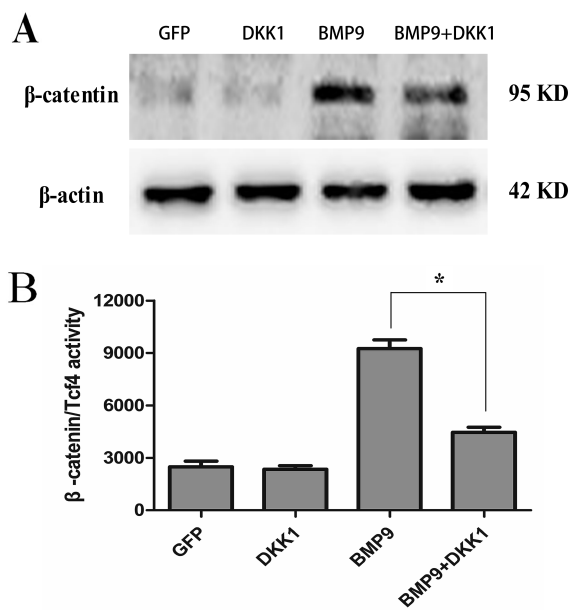

Fig. 4. Wnt/ $\beta$-catenin signaling is involved in the inhibition of BMP9-induced osteogenic differentiation by Dkk1. (A) Western blot results showed that Dkk1 inhibited the expression of $\beta$-catenin induced by BMP9 in C3H10T1/2 cells. (B) The inhibitory effect of Dkk1 on BMP-9 induced luciferase activities of pTOP-Luc reporter. At $36 \mathrm{~h}$ after infection, cells were harvested for luciferase assay. ${ }^{*} \mathrm{P}<0.01$.

teogenic differentiation by Dkk1. By Western blotting, we found that the protein level of $\beta$-catenin was increased in response to BMP9, which was significantly decreased by overexpression of Dkk1 (Fig. 4A). Therefore, BMP9 induced $\beta$-catenin/Tcf4 reporter activity, and luciferase activity of the pTOPLuc reporter, which was inhibited by exogenous Dkk1 expression (Fig. 4B). Taken together, these data indicated that Dkk1 expression inhibits the Wnt/ $\beta$-catenin signaling induced by BMP9, suggesting that $W n t / \beta$-catenin signaling is involved in the inhibition of BMP9-induced osteogenic differentiation by Dkk1. 


\section{DISCUSSION}

Mesenchymal stem cells (MSCs) are adherent marrow stromal cells, which can self-renew and have the potential to differentiate into osteoblasts, chondrocytes, myoblasts or adipocytes (26). As multi-potent progenitors, MSCs are ideal seed cells for scientific research and bone tissue engineering (27). However, promotion of MSCs differentiation into osteoblasts by osteogenic factors is a crucial issue in bone tissue engineering. Bone morphogenetic protein 9 (BMP9) is a potent inducer of osteogenic differentiation of mesenchymal stem cells (MSCs) both in vitro and in vivo $(4,5)$. In addition to BMPs, Wnt $/ \beta$-catenin signaling also plays an important role in skeletal development and bone formation $(10,11)$. Recent studies have indicated that cross-talk between these two signals is important for the regulation of osteoblast differentiation (20-22). In this study, we further characterized the role of the Wnt inhibitor Dkk1 in the cross-talk between Wnt/ $\beta$-catenin and BMP signaling in inducing osteogenic differentiation of MSCs. The findings presented here support our hypothesis that Dkk1 negatively regulates BMP9-induced osteoblast differentiation through the $\mathrm{Wnt} / \beta$-catenin pathway.

Although the molecular mechanisms underlying BMP9mediated osteogenesis remain to be fully understood, various studies have demonstrated that BMP9 plays a critical role in osteogenic differentiation (1, 3, 5, 6). Besides the direct application of recombinant BMP proteins, many reports have confirmed the ability of recombinant BMP9 adenovirus to induce bone formation both in vitro and in vivo $(1,5,6)$. Moreover, recombinant BMP9 adenovirus is an ideal vector for it could transduce osteoblast progenitor cells effectively and bioactive BMP9 is continuously produced (28). Although BMP-induced osteoblast differentiation does not need long-term expression, BMP9 adenovirus can induce osteogenic differentiation for $>2$ weeks, which is consistent with our results (Fig. 3).

Dickkopf-1 (Dkk1) is an inhibitor of Wnt signaling, which is a crucial pathway in skeletal development (15). Dkk1 is also involved in bone disease. Increased Dkk1 expression is associated with osteoarthritic cartilage chondrocyte apoptosis and rheumatoid arthritic joint disorders (29, 30), whereas overexpression of Dkk1 also plays an inportant role in osteolytic metastatic bone disease both in prostate carcinoma and multiple myeloma $(18,19)$.

In this study, we found that exogenous Dkk1 expression not only inhibited BMP9-induced ALP activity (Fig. 3A and B) but also dramatically decreased the expression of osteocalcin $(\mathrm{OCN})$ and osteopontin (OPN) and in vitro matrix mineralization by $\mathrm{C} 3 \mathrm{H} 10 \mathrm{~T} 1 / 2$ cells (Fig. 3C and D). Dkk1 is a downstream target of BMP signaling in osteoblasts (31). Osx, which is specifically expressed in all osteoblasts, is required for the differentiation of preosteoblasts into mature osteoblasts (32). Moreover, Osx is a downstream gene of Runx2, and BMPs may directly regulate Dkk1 expression through the BMPRunx2-Osx axis (33). Our study confirms that overexpression of BMP9 induces Dkk1 expression in a dose-dependent manner in MSCs (Fig. 1), suggesting that Dkk1 plays an important role in regulating the BMP9-induced osteogenic differentiation of MSCs.

The MAPK pathway is involved in BMP9-induced osteogenic differentiation of MSCs, while p38 and ERK $1 / 2$ may play different roles in regulating BMP9 osteoinductive signaling (24). In this study, the upregulation of Dkk1 expression by BMP9 was prevented by the P38 MAPK inhibitor SB203580, but was unaffected by the ERK1/2 MAPK inhibitor PD98059 (Fig. 2). This is consistent with previous reports that upregulation of Dkk1 by BMPs was blocked by P38 MAPK inhibitors both in vitro and in vivo $(31,34)$. The P38 MAPK pathway may regulate the $\mathrm{Wnt}$ signaling by BMPRIA. The Wnt inhibitor Dkk1 is a downstream target of BMP signaling through the type IA receptor, and upregulates Dkk1 expression through both Smad and non-Smad signaling (P38 MAPK) in osteoblasts. Dkk1 inhibits canonical Wnt signaling, leading to a decrease in bone mass. A high doses of BMP2 appears to reduce proliferation and increase apoptosis via Dkk1 (35).

There may be cross-talk between the BMP and Wnt pathways in inducing osteogenic differentiation of MSCs. The BMP and Wnt signaling pathways tightly regulate each other (19). Although the mechanism underlying the role of the Wnt inhibitor Dkk1 in BMP9-induced osteogenic differentiation remains to be defined, disruption of Dkk1 allows $\beta$-catenin to stimulate osteogenesis (12) and rescues dexamethasone-induced suppression of primary human osteoblast differentiation (36). $\beta$-catenin, as a key molecule in canonical Wnt signaling, may also play an important role in BMP9-induced osteogenic differentiation $(20,22)$. This was reinforced by our findings that both $\beta$-catenin expression and $\beta$-catenin/Tcf 4 activity was increased in response to BMP9, and significantly decreased by overexpression of Dkk1 (Fig. 4). Taken together, these data indicate that $\mathrm{Wnt} / \beta$-catenin signaling is involved in the inhibition of BMP9-induced osteogenic differentiation by Dkk1.

In summary, our data demonstrate that expression of the Wnt antagonist Dkk1 could be induced by BMP9 in part via the MAPK-P38 pathway. Moreover, Dkk1 dramatically decreased $\beta$-catenin and $\beta$-catenin/Tcf4 activity induced by BMP9, thereby inhibiting BMP9-induced osteogenic differentiation of MSCs. The negative feedback control effect of Dkk1 may provide an additional mechanism of crosstalk between BMPs and Wnt signaling and will enable optimization of the therapeutic use of BMP9 and for bone tissue engineering.

\section{MATERIALS AND METHODS}

\section{Cell culture and chemicals}

HEK293 and C3H10T1/2 cells were obtained from ATCC (Manassas, VA, USA). Cells were maintained in complete Dulbecco's modified Eagle's medium (DMEM, Hyclone, China), supplemented with $10 \%$ fetal bovine serum (FBS, Gibco, Australia), $100 \mathrm{U} / \mathrm{ml}$ penicillin, and $100 \mathrm{mg} / \mathrm{ml}$ strepto- 
mycin, maintained at $37^{\circ} \mathrm{C}$ in a humidified atmosphere of $5 \%$ $\mathrm{CO}_{2}$.

The MAPK inhibitors PD98059 and SB203580 were obtained from Santa Cruz (California, USA). Inhibitors were dissolved in DMSO and aliquots were stored at $-80^{\circ} \mathrm{C}$.

\section{Construction and amplification of recombinant adenoviruses expressing GFP, Dkk1, and BMP9}

Recombinant adenoviruses were generated previously using the AdEasy system (37), and subsequently used to generate recombinant adenoviruses in HEK293 cells. The resulting adenoviruses were designated AdGFP, AdDkk1, and AdBMP9 (also expressing GFP). AdGFP was used as the vector control.

\section{Alkaline phosphatase (ALP) assays}

C3H10T1/2 cells were seeded in 24-well culture plates. At 7 days after treatment, ALP activities were assessed by modified Great Escape SEAP Chemiluminescence Assay (BD Clontech, USA) (20, 25), and histochemical staining assay using the BCIP/NBT Alkaline Phosphatase Color Development Kit (Beyotime, Jiangsu, China) according to the manufacturer's instructions.

\section{Matrix mineralization detection}

$\mathrm{C} 3 \mathrm{H} 10 \mathrm{~T} 1 / 2$ cells were cultured in the presence of ascorbic acid $(50 \mathrm{mg} / \mathrm{ml}), \beta$-glycerophosphate $(10 \mathrm{mM})$, and $10^{-8} \mathrm{nM}$ dexamethasone. At 14 days after treatment, calcium deposition was analyzed by Alizarin Red-S staining (Sigma-Aldrich), as described previously (25). Briefly, cells were fixed with $4 \%$ paraformaldehyde for $10 \mathrm{~min}$ at room temperature. After being washed with distilled water, fixed cells were incubated with $2 \%$ Alizarin Red S for 30 min, followed by extensive washing with distilled water.

\section{RNA isolation and quantitative real-time PCR (qRT-PCR)}

Total RNA of the cells was isolated using TRIzol reagent (Invitrogen) according to the manufacturer's instructions. Reverse transcription reactions were performed using the PrimeScript RT reagent kit (Takara, Dalian, China). The cDNA products were further diluted fivefold and used as PCR templates. Quantitative real-time PCR was performed using SYBR Green Supermix (Bio-Rad, America) as described previously (25). The conditions maintained for real-time PCR are as follows: $95^{\circ} \mathrm{C}$ for 3 minutes for 1 cycle; $95^{\circ} \mathrm{C}$ for $10 \mathrm{sec}-$ onds, $58^{\circ} \mathrm{C}$ for 5 seconds for 40 cycles. Dissociation stage was applied at the end of the amplification procedure. Duplicate reactions were carried out for each sample, and all samples were normalized to the expression level of GAPDH. The primer sequences of Dkk1 used for qRT-PCR were as follows: the forward primer sequence for Dkk1 was 5'-CCCATGAACTCAG GTCCATT-3', and the reverse primer sequence was 5'-AAT CACTTGCTTGGGCATTC-3'.

\section{Western blotting analysis}

C3H10T1/2 cells were seeded in $100 \mathrm{~mm}$ dishes and treated for 9 days. Cell lysates were prepared using cell lysis buffer containing the protease inhibitor PMSF (Beyotime, Shanghai, China). The samples were loaded onto $10 \%$ SDS-PAGE gels. After electrophoretic separation, the proteins were transferred to PVDF membranes. The membrane was blocked with 5\% non-fat dry milk for $2 \mathrm{~h}$ and incubated at $4^{\circ} \mathrm{C}$ overnight with primary antibodies. Following this, the membrane was incubated with a secondary antibody conjugated with horseradish peroxidase. Proteins of interest were detected using an ECL kit (Beyotime Institute of Biotechnology, China). Anti-osteopontin, anti-osteocalcin, anti- $\beta$-actin, and the secondary goat and rabbit IgGs were obtained from Santa Cruz (Santa Cruz, MA). Anti- $\beta$-catenin antibody was purchased from Cell Signaling Technology (Danvers, MA). Anti-BMP9 was obtained from Abcam (Cambridge, MA).

\section{Transfection and luciferase reporter assay}

C3H10T1/2 Cells were seeded in $25 \mathrm{~cm}^{2}$ flasks and transfected with $2 \mathrm{mg}$ per flask of $\beta$-catenin/Tcf-responsive luciferase reporter, pTOP-Luc using Lipofectamine (Invitrogen) (20). At 16 $\mathrm{h}$ after transfection, cells were replated to 24-well plates and coinfected with AdGFP, AdBMP9, and/or AdDkk1. At $36 \mathrm{~h}$ after treatment, cells were lysed and subjected to luciferase assays using the Promega Luciferase Assay Kit.

\section{Statistical analysis}

Results are expressed as means \pm standard deviation (SD). All quantitative experiments were performed in triplicate and/ or repeated three times. The two-tailed Student's t-test was used to analyze statistical significance. A value of $\mathrm{P}<0.05$ was considered to indicate statistical significance.

\section{ACKNOWLEDGEMENTS}

We would like to thank Pro. Tong-Chuan He (Molecular Oncology Laboratory, Department of Surgery, University of Chicago Medical Center) for the use of AdBMP9, AdDkk1, and AdGFP. This work was supported by grants from the National Natural Science Foundation of China (No. 81372003 and No. 81171751).

\section{REFERENCES}

1. Cheng H, Jiang W, Phillips FM et al (2003) Osteogenic activity of the fourteen types of human bone morphogenetic proteins (BMPs). J Bone Joint Surg Am 85-a, 1544-1552

2. Miyazono K, Kamiya Y and Morikawa M (2010) Bone morphogenetic protein receptors and signal transduction. J Biochem 147, 35-51

3. Lamplot JD, Qin J, Nan G et al (2013) BMP9 signaling in stem cell differentiation and osteogenesis. Am J Stem Cells $2,1-21$ 
4. Song JJ, Celeste AJ, Kong FM, Jirtle RL, Rosen V and Thies RS (1995) Bone morphogenetic protein-9 binds to liver cells and stimulates proliferation. Endocrinology 136, 4293-4297

5. Luu HH, Song WX, Luo X et al (2007) Distinct roles of bone morphogenetic proteins in osteogenic differentiation of mesenchymal stem cells. J Orthop Res 25, 665-677

6. Luther G, Wagner ER, Zhu G et al (2011) BMP-9 induced osteogenic differentiation of mesenchymal stem cells: molecular mechanism and therapeutic potential. Curr Gene Ther 11, 229-240

7. Zhang Y, Xie RL, Croce CM et al (2011) A program of microRNAs controls osteogenic lineage progression by targeting transcription factor Runx2. Proc Natl Acad Sci U S A 108, 9863-9868

8. Zhao GQ (2003) Consequences of knocking out BMP signaling in the mouse. Genesis 35, 43-56

9. Wang J and Wynshaw-Boris A (2004) The canonical Wnt pathway in early mammalian embryogenesis and stem cell maintenance/differentiation. Curr Opin Genet Dev 14, 533-539

10. Clevers $H$ (2006) Wnt/beta-catenin signaling in development and disease. Cell 127, 469-480

11. Glass DA 2nd and Karsenty G (2007) In vivo analysis of Wnt signaling in bone. Endocrinology 148, 2630-2634

12. Boyden LM, Mao J, Belsky J et al (2002) High bone density due to a mutation in LDL-receptor-related protein $5 . \mathrm{N}$ Engl J Med 346, 1513-1521

13. Day TF, Guo X, Garrett-Beal L and Yang Y (2005) Wnt/beta-catenin signaling in mesenchymal progenitors controls osteoblast and chondrocyte differentiation during vertebrate skeletogenesis. Dev Cell 8, 739-750

14. Kawano Y and Kypta R (2003) Secreted antagonists of the Wnt signalling pathway. J Cell Sci 116, 2627-2634

15. Mukhopadhyay M, Shtrom S, Rodriguez-Esteban C et al (2001) Dickkopf1 is required for embryonic head induction and limb morphogenesis in the mouse. Dev Cell $1,423-434$

16. Li J, Sarosi I, Cattley RC et al (2006) Dkk1-mediated inhibition of Wnt signaling in bone results in osteopenia. Bone 39, 754-766

17. Morvan F, Boulukos K, Clement-Lacroix P et al (2006) Deletion of a single allele of the Dkk1 gene leads to an increase in bone formation and bone mass. J Bone Miner Res 21, 934-945

18. Hall CL, Bafico A, Dai J, Aaronson SA and Keller ET (2005) Prostate cancer cells promote osteoblastic bone metastases through Wnts. Cancer Res 65, 7554-7560

19. Tian E, Zhan F, Walker R et al (2003) The role of the Wnt-signaling antagonist Dkk1 in the development of osteolytic lesions in multiple myeloma. N Engl J Med 349, 2483-2494

20. Tang N, Song WX, Luo J et al (2009) BMP-9-induced osteogenic differentiation of mesenchymal progenitors requires functional canonical Wnt/beta-catenin signalling. J Cell Mol Med 13, 2448-2464

21. Kim HK, Oxendine I and Kamiya N (2013) High-concentration of BMP2 reduces cell proliferation and increases apoptosis via Dkk1 and SOST in human primary periosteal cells. Bone 54, 141-150
22. Zhang H, Wang J, Deng F et al (2015) Canonical Wnt signaling acts synergistically on BMP9-induced osteo/odontoblastic differentiation of stem cells of dental apical papilla (SCAPs). Biomaterials 39, 145-154

23. Lou J, Tu Y, Li S and Manske PR (2000) Involvement of ERK in BMP-2 induced osteoblastic differentiation of mesenchymal progenitor cell line $\mathrm{C} 3 \mathrm{H} 10 \mathrm{~T} 1 / 2$. Biochem Biophys Res Commun 268, 757-762

24. Xu DJ, Zhao YZ, Wang J, He JW, Weng YG and Luo JY (2012) Smads, p38 and ERK1/2 are involved in BMP9-induced osteogenic differentiation of C3H10T1/2 mesenchymal stem cells. BMB Rep 45, 247-252

25. Liao J, Hu N, Zhou N et al (2014) Sox9 potentiates BMP2induced chondrogenic differentiation and inhibits BMP2induced osteogenic differentiation. PLoS One 9, e89025

26. Pittenger MF, Mackay AM, Beck SC et al (1999) Multilineage potential of adult human mesenchymal stem cells. Science 284, 143-147

27. Petite H, Viateau V, Bensaid W et al (2000) Tissue-engineered bone regeneration. Nat Biotechnol 18, 959-963

28. Kang Q, Sun $\mathrm{MH}$, Cheng $\mathrm{H}$ et al (2004) Characterization of the distinct orthotopic bone-forming activity of 14 BMPs using recombinant adenovirus-mediated gene delivery. Gene Ther 11, 1312-1320

29. Diarra D, Stolina M, Polzer K et al (2007) Dickkopf-1 is a master regulator of joint remodeling. Nat Med 13, 156-163

30. Weng LH, Wang CJ, Ko JY, Sun YC, Su YS and Wang FS (2009) Inflammation induction of Dickkopf-1 mediates chondrocyte apoptosis in osteoarthritic joint. Osteoarthritis Cartilage 17, 933-943

31. Kamiya N, Kobayashi T, Mochida $Y$ et al (2010) Wnt inhibitors Dkk1 and Sost are downstream targets of BMP signaling through the type IA receptor (BMPRIA) in osteoblasts. J Bone Miner Res 25, 200-210

32. Nakashima K, Zhou X, Kunkel G et al (2002) The novel zinc finger-containing transcription factor osterix is required for osteoblast differentiation and bone formation. Cell 108, 17-29

33. Zhang C, Dai H and de Crombrugghe B (2012) Characterization of Dkk1 gene regulation by the osteoblast-specific transcription factor Osx. Biochem Biophys Res Commun 420, 782-786

34. Zuzarte-Luis V, Montero JA, Rodriguez-Leon J, Merino R, Rodriguez-Rey JC and Hurle JM (2004) A new role for BMP5 during limb development acting through the synergic activation of Smad and MAPK pathways. Dev Biol 272, 39-52

35. Zhang R, Oyajobi BO, Harris SE et al (2013) Wnt/beta-catenin signaling activates bone morphogenetic protein 2 expression in osteoblasts. Bone 52, 145-156

36. Butler JS, Queally JM, Devitt BM, Murray DW, Doran PP and O'Byrne JM (2010) Silencing Dkk1 expression rescues dexamethasone-induced suppression of primary human osteoblast differentiation. BMC Musculoskelet Disord 11, 210

37. Luo J, Deng ZL, Luo X et al (2007) A protocol for rapid generation of recombinant adenoviruses using the AdEasy system. Nat Protoc 2, 1236-1247 